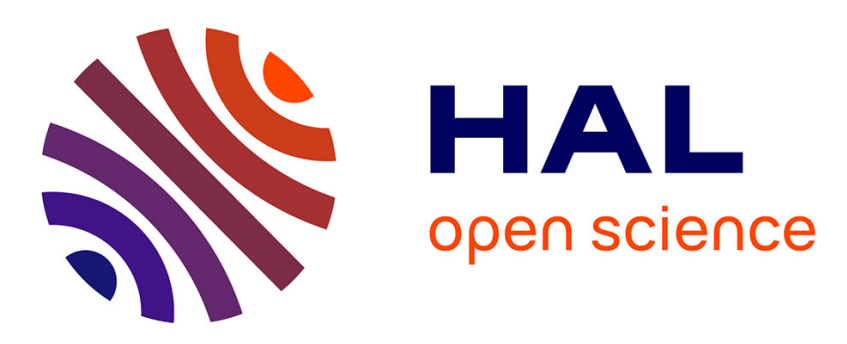

\title{
Neural-based underwater surface localization through electrolocation
}

\author{
Yannick Morel, Vincent Lebastard, Frédéric Boyer
}

\section{To cite this version:}

Yannick Morel, Vincent Lebastard, Frédéric Boyer. Neural-based underwater surface localization through electrolocation. 2016 IEEE International Conference on Robotics and Automation (ICRA), 2016, Stockholm, Sweden. pp.2596 - 2603, 10.1109/ICRA.2016.7487417 . hal-01503248

\section{HAL Id: hal-01503248 \\ https://hal.science/hal-01503248}

Submitted on 6 Apr 2017

HAL is a multi-disciplinary open access archive for the deposit and dissemination of scientific research documents, whether they are published or not. The documents may come from teaching and research institutions in France or abroad, or from public or private research centers.
L'archive ouverte pluridisciplinaire HAL, est destinée au dépôt et à la diffusion de documents scientifiques de niveau recherche, publiés ou non, émanant des établissements d'enseignement et de recherche français ou étrangers, des laboratoires publics ou privés. 


\title{
Neural-based Underwater Surface Localization through Electrolocation
}

\author{
Yannick Morel, Vincent Lebastard, and Frédéric Boyer
}

\begin{abstract}
By manipulation of electric fields, it is possible to detect the presence of foreign objects underwater. The presented work builds upon a previous result, in which was developed a neural network-based methodology allowing to address this detection problem for spherical objects. Hereafter, we show that the approach generalizes to the case of continuous walls. The technique relies on a neural model of the forward map (from scene configuration to electric measures). Exploiting this model, together with collected electric measures, it becomes possible to detect and infer the relative distance and orientation of a planar wall. In addition, we show that relying on a single forward model, only descriptive of the presence of a single wall, it is possible to address the same problem in presence of a combination of walls forming a corner or a corridor. Closing the motion control loop with information obtained using the proposed approach, it becomes possible to regulate position of a system at a fixed distance and orientation from a wall, with applications to the exploration and monitoring of flooded pipelines, or to surface quality monitoring of ships' hulls (in relation to biofouling). Data collected experimentally are used together with analytical models and numerical simulations to illustrate efficacy of the approach.
\end{abstract}

\section{INTRODUCTION}

In underwater robotics, the electric sense ([1]) is rapidly emerging as an effective complement to more classical navigation instruments, such as those based on acoustics, including SOund Navigation And Ranging systems (SONAR, [2]) and Doppler Velocity Loggers (DVL, [3]), or Inertial Navigation Systems (INS, [4]). This sensorial modality is based on manipulation of electric fields. Typically, the system applies a known electric field to its direct environment, then carefully monitors its variations. Presence of foreign objects in the system's vicinity will lead to perturbations of the emitted electric field. By detecting, measuring and interpreting these perturbations, it becomes possible to infer a range of information on the object(s) detected and more generally on the system's direct environment ([1], [5]).

The most common application of this sensing technology consists in underwater obstacle detection, localization and avoidance ([6], [7]), but a range of additional useful functionalities are achievable, including localization and movement coordination of teams of mobile robots ([8], [9]), object shape recognition ([10]), and short range communication ([11]). Such functionalities have been achieved using a

Y. Morel is with the Commissariat à l'Energie Atomique et aux Energies Renouvelables (CEA Tech Pays de la Loire), Technocampus, 44340 Bouguenais, France, ymorelegmail.com

V. Lebastard and F. Boyer are with the IRCCyN Laboratory, Mines de Nantes, 44307 Nantes, France, \{vincent.lebastard, frederic.boyer\}@mines-nantes.fr

The work presented was performed as part of the strategic partnership between CEA Tech, Mines Nantes and ARMINES, funded by the région Pays de La Loire, in the frame of the Regional Technology Transfer Platform (PRTT) of CEA Tech. wide range of processing techniques. A number of results are based on techniques inspired from those in Electric Impedance Tomography (EIT, [12]), by which one attempts to exploit available electric measures to reconstruct the impedance map of the system's near environment. In general however, such techniques require expansive sets of measures and heavy computations ([13]), and the estimation problem is oftentimes ill-conditioned ([14]). However, if instead of attempting to reconstruct a detailed impedance map, one assumes most of the environment is homogeneous and features only a limited number of foreign objects, it becomes possible to significantly alleviate the computational burden. In particular, one is then able to parameterize the scene using a relatively concise set of variables, for instance describing the objects' shape, electric properties and position in the scene. It has been shown that it is possible to reconstruct (or, more specifically: observe) these variables using electric measures together with a forward model, describing the relationship from scene geometry to achieved electric measures ([15], [16]). This scene reconstruction or estimation problem can be addressed using different approaches, including well established techniques such as Kalman filtering ([15], [16]). As an alternative to such methods, we have proposed in [17] an approach based on the development of a neural networkbased forward model ([18]). In particular, for a given type of scene (i.e. presence of a pair of cooperating mobile robots in [8], or for a spherical object in [17]), we collect series of electric measures descriptive of this scene (for a range of acceptable relative robot positions or sphere locations, respectively). Such data sets can be generated using either analytical forward models (such as that in [19]), numerical models (such as that based on the Boundary Element Method in [20], see [21] for a discussion of BEM), or an experimental setup (such as that described in [19]). Once such a model is constructed, it can be exploited to reconstruct the scene based on available electric measures. The inversion method proposed in [8] (also used in the following, in [17], and comparable to the beamforming technique presented in [22]) consists in presenting to the forward model a range of candidate scene structures, as described by the relevant variables (e.g. relative position of a robot, or location of a sphere). Then, comparing the neural model's output to the current available measure, it becomes possible to infer relative merit of different candidates. Specifically, owing to the forward map's continuity (as discussed in [8], and as illustrated by existing numerical and analytical models), and assuming that the forward neural model is faithful, outputs of the neural model for candidates close to the actual scene (as measured in terms of distance in the relevant variable space) will remain close to the available measure 


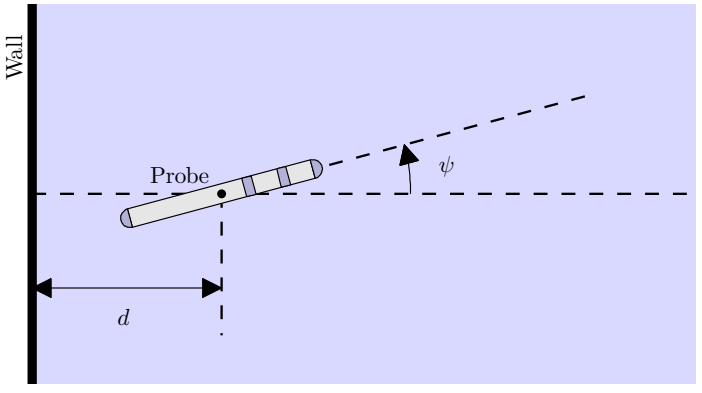

Fig. 1. Definition of scene configuration variables: distance $d$ and orientation $\psi$; wall marked as a thick black line to the left.

(distance in the measure space). Conversely, as candidate scenes become more distant from the actual one, the model outputs in general become more dissimilar to the measures. Accordingly, representing over the domain of definition of possible scenes (or of candidate scenes) the similarity or dissimilarity between corresponding model outputs and the available measure(s) allows to discriminate possible or likely candidate scenes from unlikely ones ([8], [17]).

One should note, however, that the forward map that we attempt to invert using the aforementioned procedure is not necessarily invertible (in practice, even in ideal conditions, it is only at most locally invertible due to range limitations). Invertibility depends on a number of factors, including the number of available independent measures and complexity of the scene (i.e. number of independent variables necessary to describe it). In addition, impact of the presence of a foreign object on measures rapidly decreases with distance. More specifically, the corresponding perturbation on measures typically becomes indistinguishable from measurement noise at a distance roughly equivalent to the physical length of the dipole producing the electric field (specific detection range depends on the quality of electronics used for signal acquisition, dimensions and electric properties of the object to be detected, exposed surface and material of the electrodes, and power used to produce the electric field, as discussed in [19]). Hence, once an object is far enough from the sensor, it becomes impossible to infer its situation based on electric measures. Accordingly, attempting to directly construct or identify an inverse model (from measures to scene) can be expected to prove, at the very least, problematic, if not outright impossible. Instead, the proposed approach has several benefits. In particular, precisely because it is not attempting to reconstruct the inverse map, it circumvents issues of ill-definition. Typically (as discussed in [17]), in an area of scene configuration space in which the mapping is invertible, a single scene configuration candidate will emerge as a best match (that is, when used as input to the neural model, yielding the neural output that provides the closest match to the measure available). Conversely, in a noninvertible area, such as when the sensor is to far away from the object to be detected and localized, entire swathes of the scene configuration space will emerge as equally likely. That situation makes it impossible to discriminate one single most likely location (as expected, since it is not possible to do so from available data). Yet, it remains possible to: a) come to a realization that there is ambiguity and that it is impossible to highlight a particular scene configuration candidate (in other words, we know that we do not know), b) still discriminate likely areas (instead of specific candidates) from unlikely ones. Typically, when located too far from the target object, it still remains possible to determine the area of space around the sensor within which the object is unlikely to be located, versus areas further away from the sensor where it may be.

In [17], we applied the approach delineated above to the case of a scene featuring a single spherical object. In the following, we apply the same approach to a case in which the sensor is placed in the presence of a planar, vertical wall. For ease of exposition, the problem is treated in a horizontal plane of reference, in such a manner that, assuming the considered wall is infinite, two variables are sufficient to entirely describe the scene; a distance $d \in \mathbb{R}^{+}$, and a relative orientation $\psi \in(-\pi, \pi]$ descriptive of the orientation of the sensor with respect to the wall. Definition of both variables is provided in Figure 1, which also provides a schematic view of the type of sensors considered hereafter: a slender, cylindrical electric probe composed of an insulating plastic shell (light grey in Figure 1) and of four pairs of steel electrodes (in a darker shade of grey, two hemispherical pairs at the probe's extremities, two additional cylindrical ones on the fore half). In the following, we show how, using the approach discussed in [17], we are able to capture a neural model descriptive of the relationship between a scene such as that in Figure 1 and electric measures performed by a sensor (e.g. the probe represented in this same figure). We then employ the aforementioned inversion procedure to estimate the value of $d$ and $\psi$ corresponding to a particular electric measure performed. Finally, we show that the information obtained is sufficient to close the motion control loop and allow movements of the sensor in good intelligence with its environment, including regulation of the probe's position at given distances and relative orientations from the wall.

Contribution of the presented work stems from a number of factors. In particular, the results presented illustrate the generalizability of the proposed approach, which had previously successfully been applied to solve relative positioning and coordination problems (in [8]) and sphere localization problems ([17]), and is here used to address the problem of localizing a continuous planar wall. In addition, the presented work provides a novel algorithmic tool to solve the wall localization problem in electrolocation, which had previously been addressed using for instance Kalman filters ([16]), but never using the proposed neural approach. Finally, an interesting feature of the proposed approach, differentiating it from alternative techniques, lies in its capacity of handling scenes more complex than those used to construct the direct model it relies on. Specifically, the neural model we construct is based on the presence of a single planar wall. However, it can be used in cases in which several (possibly intersecting) walls are present, forming combinations of corners and corridors, and allow safe navigation of one such scene without requiring any adjustment. By comparison, alternate approaches such as that in [16], while still able to 
handle corners and corridors, will typically require additional dedicated models.

In Section II, we provide a brief description of the experimental setup we used to collect data, present a simple analytical model used at latter stages to test the proposed approach, and discuss the neural model constructed based on data collected. Then, in Section III, we show in what manner we are able to exploit the neural model of Section II to infer position of a planar wall from performed electric measures. We also show how this same model can be used to navigate corners and corridors. Section IV concludes this paper.

\section{Experimental Setup And Collected Measures}

The problem addressed in the following is that of estimating the relative position of a mobile underwater system with respect to an insulating wall (or more generally plane surface) using electrolocation. This electrolocation approach follows a simple principle; the system, using a set of electrode located on its surface, applies an electric field to its surroundings. These same electrodes are then used to measure relevant electric variables, such as for instance difference of electric potential between electrodes (as in [23]), or electric currents flowing through the electrodes (see for instance [16]). Based on such measures, one is able to infer a range of information descriptive of the system's direct environment. In particular, presence of foreign objects, whose electric conductivity is distinct from that of the surrounding fluid, will perturb the field emitted by the system. Detecting such perturbations in the measures performed can allows to estimate a range of information regarding the foreign objects in question, including relative position in space with respect to the system, size, shape, and conductivity ([16], [23]).

\section{A. Experimental Setup}

Hereafter, we apply the neural-based approach introduced in [8] (where it is used to estimate relative position of underwater mobile systems) and later used in [17] to locate spherical objects underwater. Building upon a series of measures characterizing electric current patterns in presence of an insulating wall, it becomes possible to construct a forward model, descriptive of the relationship from scene geometry to electric measures performed. As previously alluded to, in the case considered, which is that of a single mobile system in the presence of an infinite wall, and considering motion in the horizontal plane exclusively, the scene can be described using two variables; the distance $d \in \mathbb{R}^{+}$ between wall and mobile system, and the orientation $\psi \in$ $(-\pi, \pi]$ of the mobile system with respect to the wall. The proposed approach will be tested using data collected on an experimental setup, described in detail in [19]. This setup is composed of $1 \mathrm{~m} \times 1 \mathrm{~m} \times 1 \mathrm{~m}$ glass tank filled with fresh water. The setup uses electric probes (qualitatively representing a mobile vehicle equipped with the electric sense), which are composed of a cylindrical, non-conductive plastic body (of length $22 \mathrm{~cm}, 2 \mathrm{~cm}$ diameter), and feature four pairs of steel electrodes located along the length of the probe (three pairs on the fore half, one pair aft), with each pair of electrodes following a strict left-right symmetry (probe shown in Figure 1). In practice, a $22.5 \mathrm{KHz}$ sinusoidal signal is applied to the aft-most pair of electrodes. Then, the peak-to-peak value of the current flowing through the three fore-most pairs of electrodes (connected to a common ground) is measured. These probes are mounted on a three Degree of Freedom (DoF) actuation system, allowing movement in the horizontal plane (two translations, one rotation; see [15], [19] for additional details on the experimental setup).

Position of the probe relative to a particular wall is described by the aforementioned distance $d$ and relative orientation $\psi$. Specifically, and as shown in Figure 1, we define $d$ as the distance between the probe's center and the nearest point of the wall, and assign $\psi=0$ to the relative orientation for which the probe's fore is pointing away from the wall. Below, we discuss the relationship between scene geometry, as described by $d, \psi$, and the current measures obtained by the probe. Then, data collected using the experimental setup described here is used to train a neural network providing an alternate model of the same relationship, and comparisons between experimental measures and models are performed.

\section{B. Analytical Model}

For ease of interpretation, currents measured by the probes described in Section II-A are summed and subtracted for each electrode pair. Qualitatively, looking at the sum of currents for each pair of electrodes on the probe (that is, for each pair, the sum of current flowing through the leftside and the right-side electrodes) provides insights into the position of a perturbing object in the longitudinal direction of the probe (that is, along its longitudinal axis of symmetry). Conversely, considering left-right differences (difference between the current flowing through the left-side electrode and that flowing through the right-side one) helps in determining whether objects are located port or starboard. In the following, we note the current sums $i_{\mathrm{lo}}(t) \in \mathbb{R}^{4}$, $t \geqslant 0$, where lo stands for longitudinal, and $i_{\mathrm{la}}(t) \in \mathbb{R}^{4}$, the left-right differences, where la stands for lateral. These currents can be computed as follows (see details in [19]),

$$
\begin{aligned}
& i_{\mathrm{lo}}(t)=C_{0} K(t) C_{0} u, \quad t \geqslant 0, \\
& i_{\mathrm{la}}(t)=\left(1+S^{\perp}\right) L(t) C_{0} u,
\end{aligned}
$$

where the conductance matrix $C_{0} \in \mathbb{R}^{4 \times 4}$ is a function of probe geometry and of the surrounding fluid's conductivity, the column vector $u \in \mathbb{R}^{4}$ describes the electric potentials assigned to the four pairs of electrodes, $S_{\perp} \in \mathbb{R}$ is a scaling factor, and $K(t), L(t) \in \mathbb{R}^{4 \times 4}$ are matrices computed as follows,

$$
\begin{aligned}
k_{i j} & =\frac{1}{4 \pi \gamma\left\|r_{i j}(t)\right\|}, \quad i, j=1, \ldots, 4, \quad t \geqslant 0, \\
l_{i j} & =-\frac{\bar{A}_{i}}{4 \pi\left\|r_{i j}(t)\right\|^{3}}[-\sin (\psi(t)) \quad \cos (\psi(t))] r_{i j}(t),
\end{aligned}
$$

where $k_{i j}(t), l_{i j}(t) \in \mathbb{R}$ represent the $i^{\text {th }}$ line $-j^{\text {th }}$ column entries of $K(t)$ and $L(t)$, respectively, $\gamma \in \mathbb{R}$ describes the medium's conductivity, $\bar{A}_{i} \in \mathbb{R}$ is a scaling factor function 
of the surface of the $i^{\text {th }}$ pair of electrodes, $r_{i j}(t) \in \mathbb{R}^{2}$ is the vector describing the position of the $i^{\text {th }}$ electrode pair with respect to a mirror image of the $j^{\text {th }}$ pair (specifically, the $j^{\text {th }}$ pair of electrodes of a virtual probe, mirror image of the actual one across the wall's surface, see [19]), computed as follows,

$r_{i j}(t)=\left[\begin{array}{c}2 d(t) \\ 0\end{array}\right]+\left[\begin{array}{c}\left(x_{i}+x_{j}\right) \cos (\psi(t)) \\ \left(x_{i}-x_{j}\right) \sin (\psi(t))\end{array}\right], \quad t \geqslant 0$,

where $x_{k} \in \mathbb{R}, k=1, \ldots, 4$, represents the position of electrode pair $k$ along the longitudinal axis of symmetry of the probe. Using (1)-(5), one is able to compute the value of lateral and longitudinal currents for given scene geometries, as described by values of $d(t)$ and $\psi(t)$. Finally, note that while the above model provides $i_{\mathrm{lo}}(t)$ and $i_{\mathrm{la}}(t)$, $t \geqslant 0$, for all four pairs of electrodes (meaning $i_{\mathrm{lo}}(t), i_{\mathrm{la}}(t) \in$ $\mathbb{R}^{4}$ ), due to the fact that whatever current flowing into the front three pairs is flowing out of the aft-most pair of electrode (or emitter, [19]), the overall set of measures contains information redundancy. Accordingly, it is typical to only consider measures on the front three electrode pairs, as it allows to reduce dimension of the problem with no loss of information. Therefore, in the following, we will only consider $\bar{i}_{\mathrm{lo}}(t), \bar{i}_{\mathrm{la}}(t) \in \mathbb{R}^{3}$, which describe currents flowing through the first three pairs of electrodes only.

\section{Neural Model}

We follow the approach introduced in [8], and approximate the relationship from scene geometry, described by $d, \psi$, to collected measures of the current (row) vector $i(d, \psi) \triangleq$ $\left[\bar{i}_{\mathrm{lo}}^{\mathrm{T}}(d, \psi) \quad \bar{i}_{\mathrm{la}}^{\mathrm{T}}(d, \psi)\right] \in \mathcal{D}_{i} \subset \mathbb{R}^{6}$, for a given (fixed) fluid conductivity $\gamma$, using the following neural formalism,

$$
\hat{i}(d, \psi) \triangleq \varphi(d, \psi) W, \quad(d, \psi) \in \mathcal{D}_{d} \times \mathcal{D}_{\psi} \triangleq \mathcal{D}_{\mathrm{s}},
$$

where $\hat{i}(d, \psi) \in \mathcal{D}_{i}$ provides an estimate of the current vector $i(\cdot, \cdot)$ for a scene configuration given by $d, \psi$, the row vector $\varphi(d, \psi) \in \mathbb{R}^{p}$ contains polynomial functions of $d$ and $\psi$ chosen a priori, and $W \in \mathbb{R}^{p \times 6}$ is a weight matrix, to be selected in such a manner that $\hat{i}(d, \psi)$ provides an accurate estimate of $i(d, \psi)$ over the domain of definition of admissible scenes $\mathcal{D}_{\mathrm{s}}$.

To train the above model, that is, to select an appropriate weight matrix $W$ in (6), we use sets of data representative of the scene we intend to capture. This data can be generated using either analytical (or numerical) models, such as that in Section II-B, or experimental setups such as that discussed in Section II-A. In the following, we will work using the latter: experimental data captured on the electric bench setup of Section II-A. This data was collected by adjusting the position and orientation of an electric probe with respect to one of the tank's four walls. To reduce impact of the presence of the other three walls on measures performed, movements of the probe were constrained to a line perpendicular to the wall of interest, and crossing the center of the tank. Keeping the probe between the tank's center and the wall of interest, we are able to maintain the probe at a distance greater than half a meter from other walls, which is generally sufficient to ensure the impact of their presence on measures remains largely negligible. Measures $i_{\mathrm{m}}(d, \psi) \in \mathbb{R}^{6}$ were collected for the front three pairs of electrodes (a measure $i_{\mathrm{m}}(d, \psi)$ corresponding to the actual currents $i(d, \psi) \in \mathbb{R}^{6}$ plus measurement noise). These measures were collected along straight trajectories starting at the tank's center and ending at the wall of interest. Movement speed of the probe and sampling frequency were adjusted to obtain samples about every $2 \mathrm{~mm}$ along these trajectories. The process was repeated for relative orientations of the probe with respect to the wall $\psi$ spanning $(-\pi, \pi]$ with steps of $\pi / 36 \mathrm{rad}$ ( $5 \mathrm{deg}$ ), for a total of 72 trajectories.

We used the collected data to determine an appropriate value for the weight matrix $W$. In particular, we built a current measure matrix $I_{\mathrm{m}}\left(d_{\mathrm{s}}, \psi_{\mathrm{s}}\right) \triangleq\left[\begin{array}{lll}i_{\mathrm{m}}^{\mathrm{T}}\left(d_{1}, \psi_{1}\right) & \ldots & i_{\mathrm{m}}^{\mathrm{T}}\left(d_{q}, \psi_{q}\right)\end{array}\right]^{\mathrm{T}} \in \mathbb{R}^{q \times 6}$, where $i_{\mathrm{m}}\left(d_{j}, \psi_{j}\right)$ is the current measure collected at distance $d_{j}$ from, and relative orientation $\psi_{j}$ with respect to the wall, $j=1, \ldots, q$, and $d_{\mathrm{s}}, \psi_{\mathrm{s}}$, are vectors of distances and relative orientations containing all values at which measures were collected. We then built the corresponding polynomial matrix $\Phi\left(d_{\mathrm{s}}, \psi_{\mathrm{s}}\right) \triangleq\left[\begin{array}{lll}\varphi^{\mathrm{T}}\left(d_{1}, \psi_{1}\right) & \ldots & \varphi^{\mathrm{T}}\left(d_{q}, \psi_{q}\right)\end{array}\right]^{\mathrm{T}} \in$ $\mathbb{R}^{q \times p}$, where $\varphi^{\mathrm{T}}\left(d_{j}, \psi_{j}\right)$ is a polynomial function of its inputs. Typically, we use $\varphi\left(d_{j}, \psi_{j}\right)=$ $\left[\begin{array}{lllllll}d_{j}^{0} \psi_{j}^{0} & \ldots & d_{j}^{0} \psi_{j}{ }^{k_{2}} & d_{j}^{1} \psi_{j}^{0} & \ldots & d_{j}{ }^{k_{1}} \psi_{j}{ }^{k_{2}}\end{array}\right] \in \mathbb{R}^{p}$, where $k_{1}, k_{2} \in \mathbb{N}$ are $\varphi$ 's polynomial orders and, by construction of $\varphi(\cdot, \cdot), p=\left(k_{1}+1\right) \times\left(k_{2}+1\right)$. Then, we select the neural weight matrix $W_{\mathrm{m}} \in \mathbb{R}^{p \times 6}$ corresponding to (or descriptive of) the collected set of measures as follows (computed as the most-likely, a posteriori estimate, based on collected measures, [24]),

$W_{\mathrm{m}}=\left(\Phi^{\mathrm{T}}\left(d_{\mathrm{s}}, \psi_{\mathrm{s}}\right) \Phi\left(d_{\mathrm{s}}, \psi_{\mathrm{s}}\right)+\Sigma_{\mathrm{m}}\right)^{\dagger} \Phi^{\mathrm{T}}\left(d_{\mathrm{s}}, \psi_{\mathrm{s}}\right) I_{\mathrm{m}}\left(d_{\mathrm{s}}, \psi_{\mathrm{s}}\right)$,

where the design constant $\Sigma_{\mathrm{m}} \in \mathbb{R}^{p \times p}$ allows to account for the presence of measurement noise in the data, and where explicit mention of $W_{\mathrm{m}}$ 's dependency on $\left(d_{\mathrm{s}}, \psi_{\mathrm{s}}\right)$, was omitted for brevity. Then, the captured neural model can compute predictions $\hat{i}(d, \psi)=\varphi(d, \psi) W_{\mathrm{m}}$ of current values $i(d, \psi)$, for $(d, \psi) \in[0,0.5 \mathrm{~m}] \times(-\pi, \pi]$, domain over which experimental data was collected to train the model.

To compare accuracy of the analytical model in Section II-B and of the above neural approach, we constructed one such neural model, using $k_{1}=k_{2}=12$, and $\Sigma_{\mathrm{m}}=1 \mathrm{e}^{-6} I_{\mathrm{d} p}$, where $I_{\mathrm{d} p} \in \mathbb{R}^{p \times p}$ is the $p$-dimensional identity matrix. The numerical value of parameters in the analytical model in Section II-B are $u=\left[\begin{array}{llll}0 & 0 & 0 & 10 \mathrm{~V}\end{array}\right]^{\mathrm{T}}, S^{\perp}=3.51$, $\gamma=0.04 \mathrm{~S} / \mathrm{m}, \bar{A}=\left[\begin{array}{llll}\pi / 2 & 2 & 2 & \pi / 2\end{array}\right]^{\mathrm{T}} r_{\mathrm{e}}^{2} \mathrm{~m}^{2}$, the probe radius is $r_{\mathrm{e}}=0.01 \mathrm{~m}$, positions of the electrode pairs' centers along the probe longitudinal axis of symmetry are $x=\left[\begin{array}{llll}0.105 & 0.068 & 0.03 & -0.1\end{array}\right]^{\mathrm{T}} \mathrm{m}$ (positive in the fore direction, negative aft), and the conductance matrix has the following value,

$C_{0}=\left[\begin{array}{cccc}306.146 & -126.072 & -92.715 & -87.358 \\ -124.882 & 335.734 & -127.993 & -82.858 \\ -91.705 & -128.228 & 312.126 & -92.193 \\ -87.306 & -83.591 & -93.330 & 264.229\end{array}\right] \mathrm{e}^{-5} S$. 


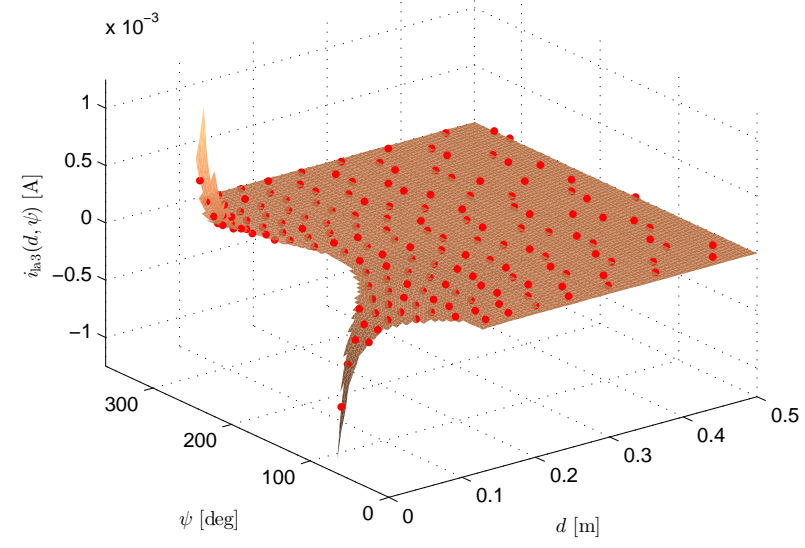

Fig. 2. Comparison between experimental data (copper surface), and output of a neural model captured using the represented data, shown with $\bullet$ 's.

We observe that, over the data set used for training, the mean error between the analytical model in Section II-B and experimental measures is $2.83 \mathrm{e}^{-2} \mathrm{~mA}$ (with a standard deviation of about $7.95 \mathrm{e}^{-2} \mathrm{~mA}$ ). Scaled by each of the six considered currents maximum amplitude (specifically, for each current in $\bar{i}_{\text {lo }}$ and $\bar{i}_{\text {la }}$ we compute the amplitude of the experimental data as the difference between maximum and minimum current value measured, we then divide the mean error obtained for each of these six currents by this amplitude to better contextualize the error), we obtain an average error of $1.003 \%$ of each current's respective maximum amplitude (standard deviation of $2.6 \%$ ). For the neural model, the mean error is of about $1.93 \mathrm{e}^{-3} \mathrm{~mA}(0.068 \%$ scaled $)$, with a standard deviation of $4.08 \mathrm{e}^{-3} \mathrm{~mA}(0.14 \%$ scaled $)$. A comparison between experimental data and output of the neural model is provided in Figure 2, where the copper surface corresponds to the collected set of data for the $3^{\text {rd }}$ lateral current (noted $\left.i_{\text {la3 }}(d, \psi)\right)$. Output of the neural model for a range of $(d, \psi)$ coordinates is marked with •'s in Figure 2. Consideration of Figure 2 gives rise to a number of points. First, the neural model appears to provide a reasonable approximation. However, such a judgement of value is fairly difficult to perform when considering Figure 2 alone. In particular, if currents' amplitude is of the order of $1 \mathrm{~mA}$ near the wall, it very rapidly drops (decreases in $1 / d^{2}$, as seen in (4)). To the extent that beyond $d=0.1 \mathrm{~m}$, it becomes difficult to discern anything as currents appear to be identically null. In reality, there remains notable and exploitable variations in currents well beyond $d=0.1 \mathrm{~m}$. To highlight that fact, we apply a logarithmic transformation to the data (as well as to the output of the neural network) as follows,

$$
\begin{array}{r}
i_{\mathrm{lj}}(d, \psi)=\frac{\operatorname{sign}\left(i_{j}(d, \psi)\right)\left(\log \left(\left|i_{j}(d, \psi)\right|+b_{j}\right)-\log \left(b_{j}\right)\right)}{\max _{(d, \psi) \in \mathcal{D}_{\mathrm{s}}}\left(\log \left(\left|i_{j}(d, \psi)\right|+b_{j}\right)-\log \left(b_{j}\right)\right)}, \\
j=1, \ldots, 6,(8)
\end{array}
$$

where $i_{j}(d, \psi)$ represents the $j^{\text {th }}$ entry of the current vector $i(d, \psi), i_{1 j}(d, \psi)$ is the logarithmically transformed current corresponding to $i_{j}(d, \psi)$, and $b_{j}$ represents an offset used to avoid the logarithmic singularity at zero. Applying this

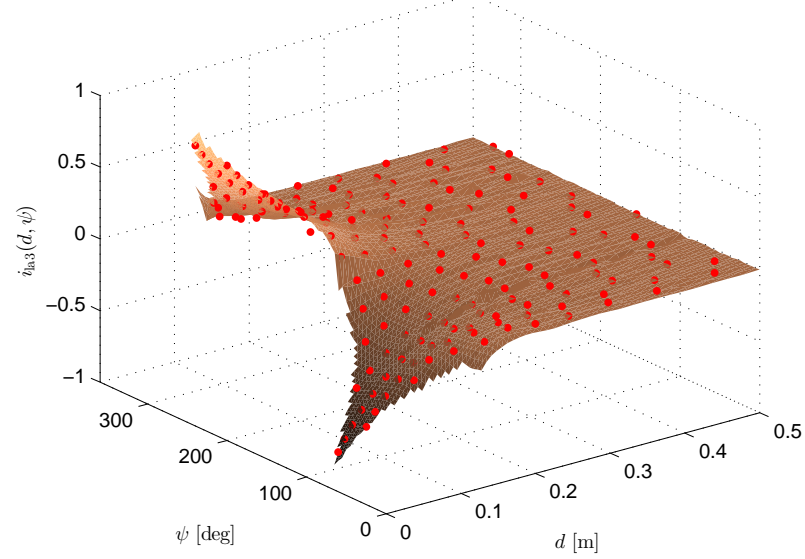

Fig. 3. Comparison between experimental data (copper surface) and neural output ( $\bullet$ 's) with logarithmic scaling.

transformation to the same data shown in Figure 2, it becomes easier to discern changes in currents at greater distance from the wall, as seen in Figure 3.

\section{WALL LOCALIZATION}

In the following, we employ the same inversion approach as that in [8], [17], which is also comparable to the beamforming technique in [22]. Specifically, we attempt to a) detect presence of a wall, and b) estimate its location and orientation relative to that of the probe. To achieve these objectives, we exploit electric measures performed by the probe together with the neural model discussed in Section IIC. In particular, when a measure of current $i_{\mathrm{m}}(d, \psi)$ is made available, we use the developed neural forward model to compute possible current measures $i_{\mathrm{c}}\left(d_{\mathrm{c}}, \psi_{\mathrm{c}}\right)$, corresponding to a range of candidate relative distances $d_{\mathrm{c}}$ and orientations $\psi_{\mathrm{c}}$. Then, comparing candidate measures $i_{\mathrm{c}}\left(d_{\mathrm{c}}, \psi_{\mathrm{c}}\right)$ to the actual measure $i_{\mathrm{m}}(d, \psi)$, it becomes possible to discriminate areas of the scene space $\mathcal{D}_{\mathrm{s}}$ likelier to contain the actual wall position $(d, \psi)$, from areas unlikely to contain it. Specifically, numerically computing the prediction error $e_{\mathrm{p}}\left(i_{\mathrm{m}}, d_{\mathrm{c}}, \psi_{\mathrm{c}}\right) \triangleq$ $\left\|i_{\mathrm{m}}-i_{\mathrm{c}}\left(d_{\mathrm{c}}, \psi_{\mathrm{c}}\right)\right\|$ over a given candidate range $\left(d_{\mathrm{c}}, \psi_{\mathrm{c}}\right) \in$ $\mathcal{D}_{\mathrm{c}} \subset \mathcal{D}_{\mathrm{s}}$, areas of $\mathcal{D}_{\mathrm{c}}$ featuring (relatively) high values of $e_{\mathrm{p}}$ are less likely to contain the actual wall position, whereas areas featuring lower error are more likely to contain it.

\section{A. Wall Localization over Open-loop Probe Trajectory}

To test the proposed approach, we consider a case in which the probe is in the presence of a single wall. The probe is made to move along a quarter circle. Trajectory of the probe's center is represented with a thin blue line in Figure 4 , with an initial position marked with an $\times$, and a final position with $a \diamond$. The shape of the probe itself is also shown in its final position. The wall is marked with a thick black line (along the $x=0$ line). Orientation of the probe in its initial position is such that it is pointing towards the wall $(\psi=\pi \mathrm{rad}$, in accordance with Figure 1$)$, and is made to continuously decrease to reach $\psi=\pi / 2 \mathrm{rad}$ at the final position (probe parallel to the wall, as shown in 


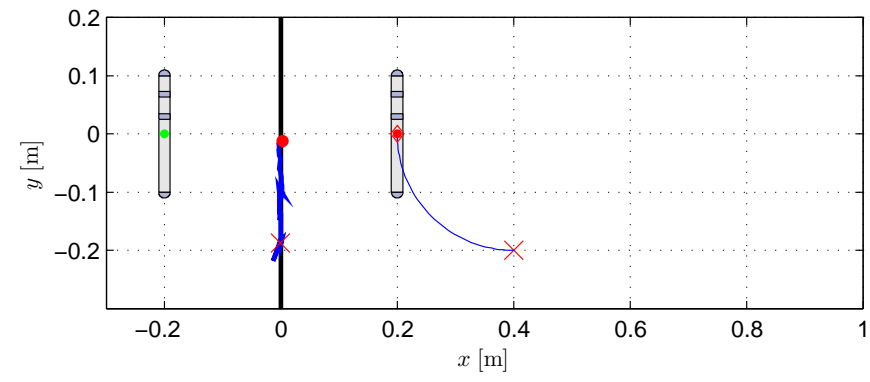

Fig. 4. Electric probe moving in an open-loop trajectory; probe trajectory represented with a thin blue line, trajectory of the wall position estimate shown with a thick blue line, initial probe position marked with an $\times$, final position with $\mathrm{a} \diamond$, final position of the probe reflection marked with a $\bullet$.

Figure 4), in such a manner that the probe's longitudinal axis of symmetry remains tangent to the $x y$-trajectory of the probe's center. To estimate position of the wall, we used the forward neural model discussed in Section II. Model inversion was performed using the iterative two-step procedure presented in [8]. In a first step, we compute predicted current measures on a grid of candidate positions $\left(d_{\mathrm{c}}, \psi_{\mathrm{c}}\right) \in\left[1 e^{-2} \mathrm{~m}, 0.4 \mathrm{~m}\right] \times[0,2 \pi) \triangleq \mathcal{D} \mathrm{c} 1$, with steps of $5 \mathrm{~cm}$ in the $d$ direction and $\pi / 12 \mathrm{rad}$ in the $\psi$ direction. A first estimate of the wall's relative position $\left(\hat{d}_{1}, \hat{\psi}_{1}\right)$ is selected as the candidate position for which the current prediction error is minimal over the candidate grid; that is,

$$
(\hat{d}, \hat{\psi})=\underset{\left(d_{\mathrm{c}}, \psi_{\mathrm{c}}\right) \in \mathcal{D}_{\mathrm{c}}}{\operatorname{argmin}} e_{\mathrm{p}}\left(i_{\mathrm{m}}, d_{\mathrm{c}}, \psi_{\mathrm{c}}\right)
$$

Then, with use a finer candidate grid, centered about the previous estimate $\left(\hat{d}_{1}, \hat{\psi}_{1}\right)$, with a grid width of $0.1 \mathrm{~m}$ in $d$ and $\pi / 12$ rad in $\psi$, and grid steps of $2.5 \mathrm{e}^{-3} \mathrm{~m}$ in $d$ and $\pi / 180 \mathrm{rad}$ in $\psi$. This second grid's candidate with lowest prediction error is selected as the estimated relative position of the wall. We represented this estimated position as a thick blue line in Figure 4, with initial estimated wall position marked with an $\times$, and the final one with a $\bullet$. The current measures used to estimate position of the wall as shown in Figure 4 were obtained using the reflection model in Section II-B (note the probe's final position reflection, represented to the left of the wall in Figure 4, with a marking its center). Reconstructing the estimated wall (closest with respect to the probe) position (in thick blue in Figure 4), and comparing it to its actual (closest) position over the quarter circle trajectory considered, we obtain an average position error of about $7 \mathrm{e}^{-3} \mathrm{~m}$ with standard deviation $6.2 \mathrm{e}^{-3} \mathrm{~m}$. In other words, and as is apparent from Figure 4, the approach is able to reconstruct the wall's position, based on the neural model and provided current measures, with reasonable accuracy. The reconstruction error for the final probe position is shown in Figure 5, computed over a wide range of candidate distances and orientations. The resulting surface's lowest point designates the most likely candidate position. Trajectory of this estimate in the $d-\psi$ plane is shown in blue; the actual relative position is in green.

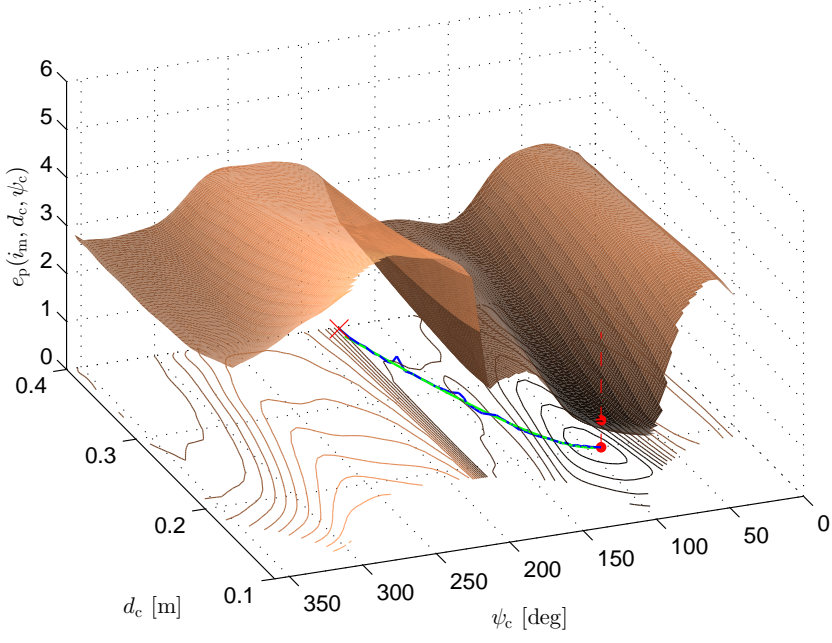

Fig. 5. Error surface for the final probe position, the surface's minimum represents the most likely candidate position and is selected as an estimate of this position. This estimate's trajectory is shown as a blue line, whereas the actual wall relative position is shown in a green line.

\section{B. Closing the Motion Control Loop}

Exploiting the information provided by the wall position estimate, it becomes possible to guide the probe's movements in such a manner that it avoids colliding with the wall, or even remains constrained at a consistent distance from this wall. More specifically, we assign the probe simple nonholonomic kinematics of the usual form,

$$
\begin{aligned}
\dot{x}(t) & =v(t) \cos \left(\psi_{\mathrm{a}}(t)\right), & x(0) & =x_{0}, \quad t \geqslant 0, \\
\dot{y}(t) & =v(t) \sin \left(\psi_{\mathrm{a}}(t)\right), & y(0) & =y_{0}, \\
\dot{\psi}_{\mathrm{a}}(t) & =\omega(t), & \psi_{\mathrm{a}}(0) & =\psi_{\mathrm{a} 0},
\end{aligned}
$$

where $x(t), y(t) \in \mathbb{R}$, represent the probe's center position, $\psi_{\mathrm{a}} \in(-\pi, \pi]$ its orientation with respect to the $x y$-frame of reference (with $\psi_{\mathrm{a}}=0$ when the probe points towards the $x$-axis' positive direction), $v(t), \omega(t) \in \mathbb{R}$, are the longitudinal displacement speed and angular velocity, which act here as control inputs to system (10)-(12). These inputs are computed as the sum of two terms, $v(t)=v_{\mathrm{c}}(t)+v_{\mathrm{j}}(t)$, $\omega(t)=\omega_{\mathrm{c}}(t)+\omega_{\mathrm{j}}(t), t \geqslant 0$, where $v_{\mathrm{j}}(t), \omega_{\mathrm{j}}(t) \in \mathbb{R}$, are obtained from a joystick's output, allowing the user to direct movements of the probe, whereas $v_{\mathrm{a}}(t), \omega_{\mathrm{a}}(t) \in \mathbb{R}$, are computed using wall position estimates as follows,

$$
\begin{array}{r}
v_{\mathrm{c}}(t)=\min \left(\max \left(\left|\hat{d}(t)-d_{\mathrm{d}}\right|-d_{\mathrm{t}}, 0\right), v_{\mathrm{s}}\right), \quad t \geqslant 0, \\
\omega_{\mathrm{c}}(t)=g_{\omega}\left(\operatorname{sign}\left(v_{\mathrm{j}}(t) \delta_{\psi}(t)\right) \psi_{\mathrm{dm}} \tanh \left(\frac{\hat{d}(t)-d_{\mathrm{d}}}{d_{\mathrm{d}}} \pi\right)\right. \\
\left.+\delta_{\psi}(t)-\operatorname{sign}\left(\delta_{\psi}(t)\right) \pi / 2\right)
\end{array}
$$

where $v_{\mathrm{s}}=0.1 \mathrm{~m} / \mathrm{s}$ represents the maximum assignable velocity, $d_{\mathrm{d}}=0.1 \mathrm{~m}$ is the distance from the wall at which we intend to regulate the probe, $d_{\mathrm{t}}=0.01 \mathrm{~m}$ is a distance threshold, $\delta_{\psi}(t) \triangleq \pi-\hat{\psi}(t)$ describes orientation of the probe with respect to the wall, $\psi_{\mathrm{dm}}=\pi / 4$ represents a maximum desired relative heading, $g_{\omega}=0.2$ is a control gain, and $\operatorname{sign}(\cdot)$ is the usual signum function. Value of 

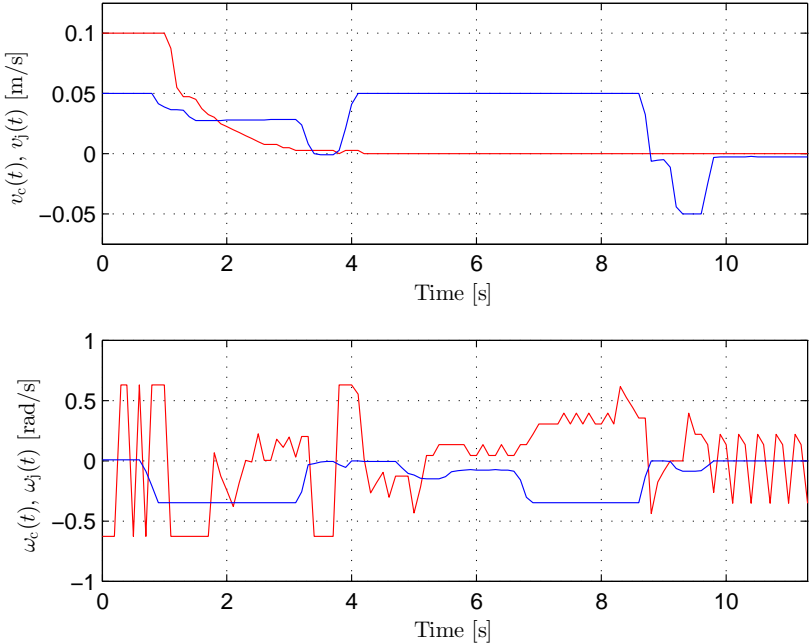

Fig. 6. Control signals, $v_{\mathrm{j}}(t), \omega_{\mathrm{j}}(t), t \geqslant 0$, shown in blue, $v_{\mathrm{C}}(t), \omega_{\mathrm{c}}(t)$, in red.

$v_{\mathrm{c}}(t)$ is by construction saturated at $v_{\mathrm{s}}=0.1 \mathrm{~m} / \mathrm{s}$, whereas we saturate the value computed by (14) at $\pi / 5 \mathrm{rad} / \mathrm{s}$. In addition, value of $v_{\mathrm{j}}(t)$ and $\omega_{\mathrm{j}}(t)$ is obtained by scaling the joystick's output signals on the front-back and left-right axes from 0 with the stick at the center, up to $0.05 \mathrm{~m} / \mathrm{s}$ and $0.35 \mathrm{rad} / \mathrm{s}$ at maximum tilt on either axis, respectively. The closed-loop is simulated using MathWorks ${ }^{\circledR}$ Simulink ${ }^{\circledR}$, together with a USB joystick. The neural model and inversion procedures are the same as in Section III-A, the electrical model used to produce electric measures is that in Section II-B. A wall is placed parallel to the $y$-axis at $x=-0.5 \mathrm{~m}$, the probe is initially located at $(x, y)=(-0.2 \mathrm{~m},-0.2 \mathrm{~m})$ with orientation $\psi_{\mathrm{a}}=\pi \mathrm{rad}$. Control signals are shown in Figure 6 ; the corresponding probe trajectory is represented in blue in Figure 7. Initially, the user is providing a positive velocity command (in blue in Figure 6, up). The regulating command (13) (in red, up) is also pushing the probe forward, until it reaches the desired distance from the wall $d_{\mathrm{d}}=0.1 \mathrm{~m}$. Once the probe has reached its desired position with respect to the wall (at fixed relative distance and orientation), the command (13)-(14) is able to maintain this desired position, using only estimates of distance and orientation provided by the electric measures and estimation algorithm discussed above. At around $8 \mathrm{~s}$, the user is attempting to wrestle orientation of the probe away from its regulated value. However, the regulating signal compensates and is able to maintain the orientation at a value near the desired one, as seen in the probe last position shown in Figure 7. Movements parallel to the wall (along the green line in Figure 7)

Finally, we reproduce the same simulation, using the same neural forward model, descriptive of the presence of a single planar wall, but now place the probe in presence of combinations of walls, forming either a corner or a corridor. We use the same control inputs (13)-(14) to regulate distance from the wall and orientation of the probe. Results are shown in Figure 8 and Figure 9. In both cases, the estimation procedure is able to reasonably well estimate position of (the closest) wall. In the case of the corner, distance is

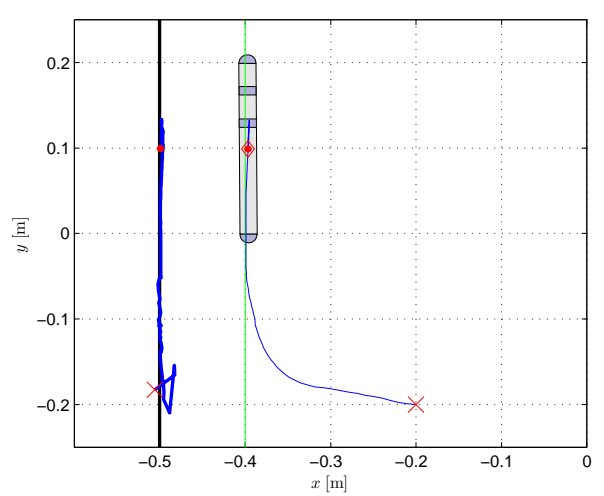

Fig. 7. Closed-loop probe trajectory, with virtual constraint at $0.1 \mathrm{~m}$ from the wall.

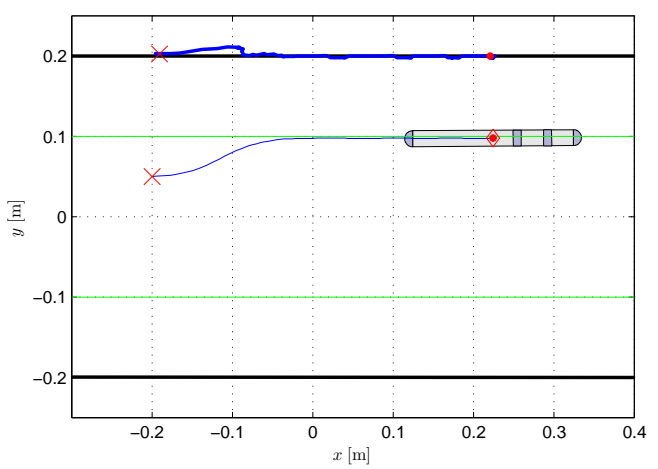

Fig. 8. Closed-loop probe trajectory in a corridor.

underestimated in a given area due to the concurrent (and significant) influence of both walls on measures. Nevertheless, the method generalizes well in that it is capable of safely regimenting movements of the probe along the walls. Note in addition that it did not require new or additional modeling considerations to account for the presence of several walls.

\section{CONCLUSION}

In the work presented, we have applied the electrolocation algorithm (first introduced in [8]) to estimate the relative distance and orientation of an underwater planar surface from an electric sensor. The approach is based on the development of a neural forward model, describing the map from scene geometry to electric measures. This neural network can be trained using either experimental data (as was the case here), or numerical data obtained from analytical of numerical models. To invert this forward model and estimate scenerelative information from measures, a series of candidate relative wall positions are fed to the neural network. Output for each candidate is then compared to the available electric measure. Based on similarity between model outputs and measures, it becomes possible to discriminate likely scene configurations from unlikely one and, in fine, reach an estimate. The approach was shown to work using a forward model obtained from experimental data. Numerical simulation results show that the information reconstructed is sufficient to enforce virtual movement constraints on a 


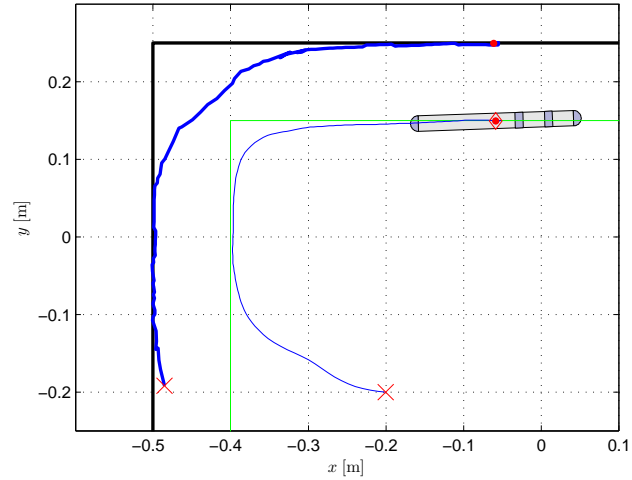

Fig. 9. Closed-loop probe trajectory in a corner.

system and regulate its distance and orientation with respect to the detected wall (while leaving the degree of freedom of displacement along the wall free). Using a single forward model descriptive of the presence of a single wall, it is possible to successfully enforce such virtual constraints for combinations of walls, including corners and corridors. Further generalization of the proposed approach to localize non-planar walls (slanted, curved, or of arbitrary shape) is ongoing.

\section{REFERENCES}

[1] I. D. Neveln, Y. Bai, J. B. Snyder, J. R. Solberg, O. M. Curet, K. M. Lynch, and M. A. MacIver, "Biomimetic and bio-inspired robotics in electric fish research," The Journal of Experimental Biology, vol. 216, no. 13, pp. 2501-2514, 2013.

[2] W. C. Knight, R. G. Pridham, and S. M. Kay, "Digital signal processing for SONAR," Proc. of the IEEE, vol. 69, no. 11, pp. 1451-1506, 1981.

[3] M. B. Larsen, "High performance doppler-inertial navigation: Experimental results," in MTS/IEEE Conf. and Exh. OCEANS, vol. 2, pp. 1449-1456, 2000.

[4] A. C. Woolsey, "Review of marine control systems: Guidance, navigation, and control of ships, rigs and underwater vehicles," Journ. of Guidance, Control, and Dynamics, vol. 28, no. 3, pp. 574-575, 2005.

[5] M. McIver and M. Nelson, "Towards a biorobotic electrosensory system," Aut. Rob., vol. 11, 2001.

[6] F. Boyer, V. Lebastard, C. Chevallereau, and N. Servagent, "Underwater reflex navigation in confined environment based on electric sense," IEEE Trans. on Robotics, vol. 29, no. 4, pp. 945-956, 2013.

[7] K. D. Dimble, B. N. Ranganathan, J. Keshavan, and J. S. Humbert, "Computationally efficient underwater navigational strategy in electrically heterogeneous environments using electrolocation," in Proc. 2015 IEEE Int. Conf. on Rob. and Aut., (Seattle, WA), pp. 11721177, 2015.

[8] Y. Morel, M. Porez, and A. J. Ijspeert, "Estimation of relative position and coordination of mobile underwater robotic platforms through electric sensing," in IEEE Int. Conf. on Robotics and Automation (ICRA), pp. 11311136, 2012.
[9] C. Chevallereau, F. Boyer, V. Lebastard, and M. Benachenou, "Electric sensor based control for underwater multi-agents navigation in formation," in Proc. 2012 IEEE Int. Conf. on Rob. and Aut., (St. Paul, MN), 2012.

[10] F. Boyer and V. Lebastard, "Exploration of objects by an underwater robot with electric sense," in Biomimetic and Biohybrid Systems, pp. 50-61, 2012.

[11] W. Wang, J. Zhao, W. Xiong, F. Cao, and G. Xie, "Underwater electric current communication of robotic fish: Design and experimental results," in Proc. 2015 IEEE Int. Conf. on Rob. and Aut., (Seattle, WA), pp. 1166-1171, 2015.

[12] J. Snyder, Y. Silverman, Y. Bai, and M. A. MacIver, "Underwater object tracking using electrical impedance tomography," in 2012 IEEE/RSJ Int. Conf. on Intelligent Robots and Systems (IROS), pp. 520-525, 2012.

[13] M. Cheney, D. Isaacson, and J. C. Newell, "Electrical impedance tomography," SIAM review, vol. 41, no. 1, pp. 85-101, 1999.

[14] A. P. Calderón, "On an inverse boundary value problem,” Comp. Appl. Math, vol. 25, no. 2-3, 2006.

[15] V. Lebastard, C. Chevallereau, A. Girin, F. Boyer, and P.-B. Gossiaux, "Localization of small objects with electric sense based on Kalman filter," in IEEE Int. Conf. on Robotics and Automation (ICRA), pp. 1137 1142, 2012.

[16] V. Lebastard, C. Chevallereau, A. Girin, N. Servagent, P. B. Gossiaux, and F. Boyer, "Environment reconstruction and navigation with electric sense based on a Kalman filter," The International Journal of Robotics Research (IJRR), vol. 32, no. 2, pp. 172-188, 2013.

[17] Y. Morel, V. Lebastard, and F. Boyer, "Neural-based underwater spherical object localization through electrolocation," in IEEE Int. Conf. on Robotics and Automation (ICRA), pp. 23-28, 2015.

[18] S. Haykin, Neural Networks: A Comprehensive Foundation. Upper Saddle River, NJ: Prentice Hall, 2004.

[19] F. Boyer, P.-B. Gossiaux, B. Jawad, V. Lebastard, and M. Porez, "Model for a sensor inspired by electric fish," IEEE Trans. on Robotics, vol. 28, no. 2, pp. 492-505, 2012.

[20] M. Porez, V. Lebastard, A. J. Ijspeert, and F. Boyer, "Multi-physics model of an electric fish-like robot: Numerical aspects and application to obstacle avoidance," in Proc. 2011 IEEE/RSJ Int. Conf. on Intelligent Robots and Systems, (San Francisco, CA), 2011.

[21] C. Liu, Y. Chen, J. Zhang, and Q. Chen, "CPG driven locomotion control of quadruped robot," in Proc. 2009 IEEE Int. Conf. on Systems, Man, and Cybernetics, (San Antonio, TX), pp. 2368-2373, 2009.

[22] N. Nguyen, I. Wiegand, and D. L. Jones, "Sparse beamforming for active underwater electrolocation," in IEEE Int. conf. on Acoustics, Speech and Signal Processing, (Taipei, Taiwan), pp. 2033-2036, 2009.

[23] Y. Bai, J. B. Snyder, M. Peshkin, and M. A. MacIver, "Finding and identifying simple objects underwater with active electrosense," The Int. Journ. of Robotics Research, 2015.

[24] C. E. Rasmussen, Gaussian Processes for Machine Learning. Cambridge, MA: MIT Press, 2006. 